\title{
Quantitative Assessment of Interim PET/CT Could Have More Prognostic Relevance than Visual Assessment for Predicting Clinical Outcome of Extranodal Diffuse Large B Cell Lymphoma
}

\author{
DONG WON BAEK ${ }^{1}$, HEE JEONG CHO ${ }^{1}$, JU-HYUNG KIM ${ }^{1}$, SANG KYUN SOHN ${ }^{1}$, GA-YOUNG SONG ${ }^{2}$, \\ SEO-YEON AHN ${ }^{2}$, SUNG-HOON JUNG ${ }^{2}$, JAE SOOK AHN ${ }^{2}$, JE-JUNG LEE ${ }^{2}$, HYEOUNG-JOON KIM ${ }^{2}$, \\ SHIN-YOUNG JEONG ${ }^{3}$, CHAE MOON HONG ${ }^{3}$, JUNG-JOON MIN ${ }^{4}$, JOON-HO MOON ${ }^{1}$ and DEOK-HWAN YANG ${ }^{2}$ \\ ${ }^{1}$ Department of Hematology/Oncology, Kyungpook National University Hospital, \\ School of Medicine, Kyungpook National University, Daegu, Republic of Korea; \\ ${ }^{2}$ Department of Hematology-Oncology, Chonnam National University Hwasun Hospital, \\ School of Medicine, Chonnam National University, Hwasun, Republic of Korea; \\ ${ }^{3}$ Department of Nuclear Medicine, Kyungpook National University Hospital, \\ School of Medicine, Kyungpook National University, Daegu, Republic of Korea; \\ ${ }^{4}$ Department of Nuclear Medicine, Chonnam National University Hwasun Hospital, \\ School of Medicine, Chonnam National University, Hwasun, Republic of Korea
}

\begin{abstract}
Background/Aim: The present study retrospectively investigated the predictive accuracy of interim positron emission tomography/computed tomography (iPET/CT) based on the Deauville 5-point scale (5-PS) and a quantitative SUVbased assessment in patients with extranodal (EN) diffuse large B cell lymphoma (DLBCL). Patients and Methods: The Deauville 5-PS and the SUVmax reduction ( $\triangle S U V \max$ ) assessment for interpreting the response to $i P E T / C T$ were used. Results: A total of 163 patients were enrolled in this study. With a median follow-up of 52.5 months, $\Delta S U V \max$ successfully predicted the survival outcomes of patients with one extranodal (EN) involvement in terms of overall survival
\end{abstract}

This article is freely accessible online.

Correspondence to: Joon Ho Moon, MD, Ph.D., Department of Hematology/Oncology, Kyungpook National University Hospital, School of Medicine, Kyungpook National University, 130 Dongdeok-ro, Jung-gu, Daegu, 41944, Republic of Korea. Tel: +82 532006314, Fax: +82 534262046, e-mail: jhmoon@knu.ac.kr; Deok-Hwan Yang, MD, Ph.D., Department of Hematology/ Oncology, Chonnam National University Hwasun Hospital, School of Medicine, Chonnam National University, 322 Seoyang-ro, Hwasun-eup, Hwasun-gun, Jeollanam-do, 58128, Republic of Korea. Tel: +82 613797636, Fax: +82 613797628, e-mail: drydh1685@hotmail.com

Key Words: DLBCL, extranodal, interim PET/CT, SUV, Deauville 5-point scale.
(OS) ( $p=0.012)$ and progression-free survival (PFS) $(p<0.001)$. Visual assessment using the Deauville 5-PS did not predict survival outcomes in patients with one or more EN involvements in terms of $O S$ and PFS. Conclusion: The quantitative SUV-based assessment with iPET/CT was a significant prognosticator for long-term survival outcomes, especially in patients with one EN involvement.

Diffuse large B cell lymphoma (DLBCL) is the most common type of non-Hodgkin lymphoma (NHL) worldwide (1). Clinically, most patients present with a rapidly growing tumor mass involving one or more lymph nodes, and approximately $40 \%$ of patients have extranodal (EN) disease (2). With the advent of the anti-CD20 monoclonal antibody, R-CHOP (Rituximab, cyclophosphamide, doxorubicin, vincristine, prednisolone) combination chemotherapy has improved response rates and overall survival in patients with $\operatorname{DLBCL}(3,4)$.

${ }^{18} \mathrm{~F}$-fluorodeoxyglucose (FDG) positron emission tomography/computed tomography (PET/CT) has emerged as a powerful functional imaging tool for staging and response assessment of malignant lymphoma (5-7). New guidelines were presented for incorporating PET/CT into standardized response criteria by the International Working Group (IWG) in 2007 (7). Furthermore, interim PET/CT (iPET/CT) has been used to evaluate the therapeutic efficacy and predict long-term outcomes at early time points. However, the predictive value of iPET/CT has been inconsistent in some studies (8-10). In an attempt to 
standardize the reporting criteria for iPET/CT, the First International Workshop on interim-PET scan in lymphoma suggested the visual response criteria using the Deauville five-point scale (5-PS) (11). In addition, the reduction rate of the maximum standardized uptake value $(\triangle S U V \max )$ was also introduced by several studies to decrease the frequency of false-positives and inter-observer variability in interpretations $(12,13)$.

It is widely accepted that DLBCL with EN involvements is less likely to be cured by standard R-CHOP therapy $(14,15)$. Therefore, early response assessment during the first-line treatment is important to improve clinical outcomes in DLBCL with EN involvements. Although recent studies have demonstrated that PET/CT generally detects EN sites more accurately than conventional CT in lymphomas $(16,17)$, challenges remain in the assessment of $\mathrm{iPET} / \mathrm{CT}$ response for EN DLBCL. As FDG is not a tumor-specific substance, it may accumulate in anatomic sites in various benign conditions, which could give rise to false positives, particularly in iPET/CT assessments of patients with multifocal, noncontiguous involvement at EN sites (18). The pitfalls of iPET/CT interpretation in EN DLBCL might be related to the definition of response without the concern of inflammatory physiology or anatomic variations (19-21). Furthermore, several studies have reported that iPET/CT during R-CHOP therapy has poor prognostic significance, possibly due to false determinations by visual assessment methods (22-24).

Accordingly, the purpose of this study is to investigate the predictive accuracy of the iPET/CT response based on visual and quantitative SUV-based assessments in DLBCL patients with EN involvements. In addition, we compared the clinical significance of Deauville 5-PS and quantitative SUV-based assessment for iPET/CT response evaluation to establish an optimal treatment strategy for EN DLBCL.

\section{Patients and Methods}

Patients. Patients with newly diagnosed DLBCL and one or more EN involvements between May 2005 and May 2014 from two independent institutions were retrospectively investigated. Patients who had central nervous system involvement or were diagnosed with only nodal involvements were excluded. All patients had an initial PET/CT at diagnosis and a subsequent iPET/CT during RCHOP chemotherapy. This study was approved by the Institutional Review Board of each participating center.

Treatment and response evaluation. Patients were treated with six to eight cycles of R-CHOP chemotherapy in standard doses (rituximab $375 \mathrm{mg} / \mathrm{m}^{2}$ i.v. on day 1 (D1), cyclophosphamide 750 $\mathrm{mg} / \mathrm{m}^{2}$ i.v. on D1, vincristine $1.4 \mathrm{mg} / \mathrm{m}^{2}$ i.v. on D1, doxorubicin 50 $\mathrm{mg} / \mathrm{m}^{2}$ i.v. on D1, and prednisolone $60 \mathrm{mg} / \mathrm{m}^{2}$ p.o. on days $1-5$ ) every 3 weeks) $(4,25)$. Three response evaluations, including PET/CT, were conducted: the first after three cycles (for patients whose plan was treatment with six cycles of primary chemotherapy) or four cycles (for patients whose plan was treatment with eight
Table I. Patient characteristics.

\begin{tabular}{lc}
\hline Variable & $\mathrm{N}(\%)$ \\
\hline Number of patients & 163 \\
Age, median years (range) & $61(18-83)$ \\
Gender & \\
$\quad$ Male & $93(57.1 \%)$ \\
Female & $70(42.9 \%)$ \\
ECOG Performance status & \\
$0-1$ & $140(85.9 \%)$ \\
$2-3$ & $23(14.1 \%)$ \\
Ann Arbor stage & \\
I-II & $75(46.0 \%)$ \\
III-IV & $88(54.0 \%)$ \\
Increased LDH & $82(50.3 \%)$ \\
Bone marrow involvement & $22(13.5 \%)$ \\
Number of extranodal sites & \\
1 & $102(62.6 \%)$ \\
2 & $39(23.9 \%)$ \\
3 & $18(11.0 \%)$ \\
4 & $4(2.5 \%)$ \\
IPI risk group & \\
Low & $63(38.7 \%)$ \\
Low-intermediate & $32(19.6 \%)$ \\
High-intermediate & $29(17.8 \%)$ \\
High & $39(23.9 \%)$ \\
NCCN-IPI & \\
Low & $11(6.4 \%)$ \\
Low-intermediate & $73(42.7 \%)$ \\
High-intermediate & $69(40.4 \%)$ \\
Autoh & $18(10.5 \%)$ \\
Relapse & $22(13.5 \%)$ \\
Death & $40(23.4 \%)$ \\
\hline
\end{tabular}

ECOG: Eastern Cooperative Oncology Group; IPI: International Prognostic Index; LDH: lactate dehydrogenase; NCCN: National Comprehensive Cancer Network; SCT: stem cell transplantation.

cycles of primary chemotherapy) of R-CHOP and the second after primary chemotherapy. The third response evaluation was conducted within a month of completing the first-line chemotherapy. Followup restaging was conducted every three or six months during the first year after first-line chemotherapy, and every six months thereafter. The responses were assessed according to the revised International Workshop Criteria (IWC) (7).

${ }^{18}$ F-FDG PET/CT and image analysis. All patients underwent $\mathrm{PET} / \mathrm{CT}$ imaging on a Discovery ST PET/CT system (GE Healthcare, Chicago, IL, USA), consisting of a bismuth germanate full scanner and a 16-detector-row computed tomography (CT) scanner. Patients fasted for at least $6 \mathrm{~h}$ prior to the intravenous administration of FDG (7.4 megabecquerel $(\mathrm{MBq})$ per $\mathrm{kg}$ of body weight) to ensure a serum glucose level below $7.2 \mathrm{mmol} / \mathrm{l}$. One hour after FDG administration, a low-dose CT scan was obtained without contrast enhancement for attenuation correction $(120 \mathrm{kV}$, automated from 10 to $130 \mathrm{~mA}$, a $512 \times 512$ matrix, a $50-\mathrm{cm}$ field of view (FOV), 3.75-mm slice thickness), extending from the base of the skull to the proximal thighs. Immediately after CT acquisition, PET 

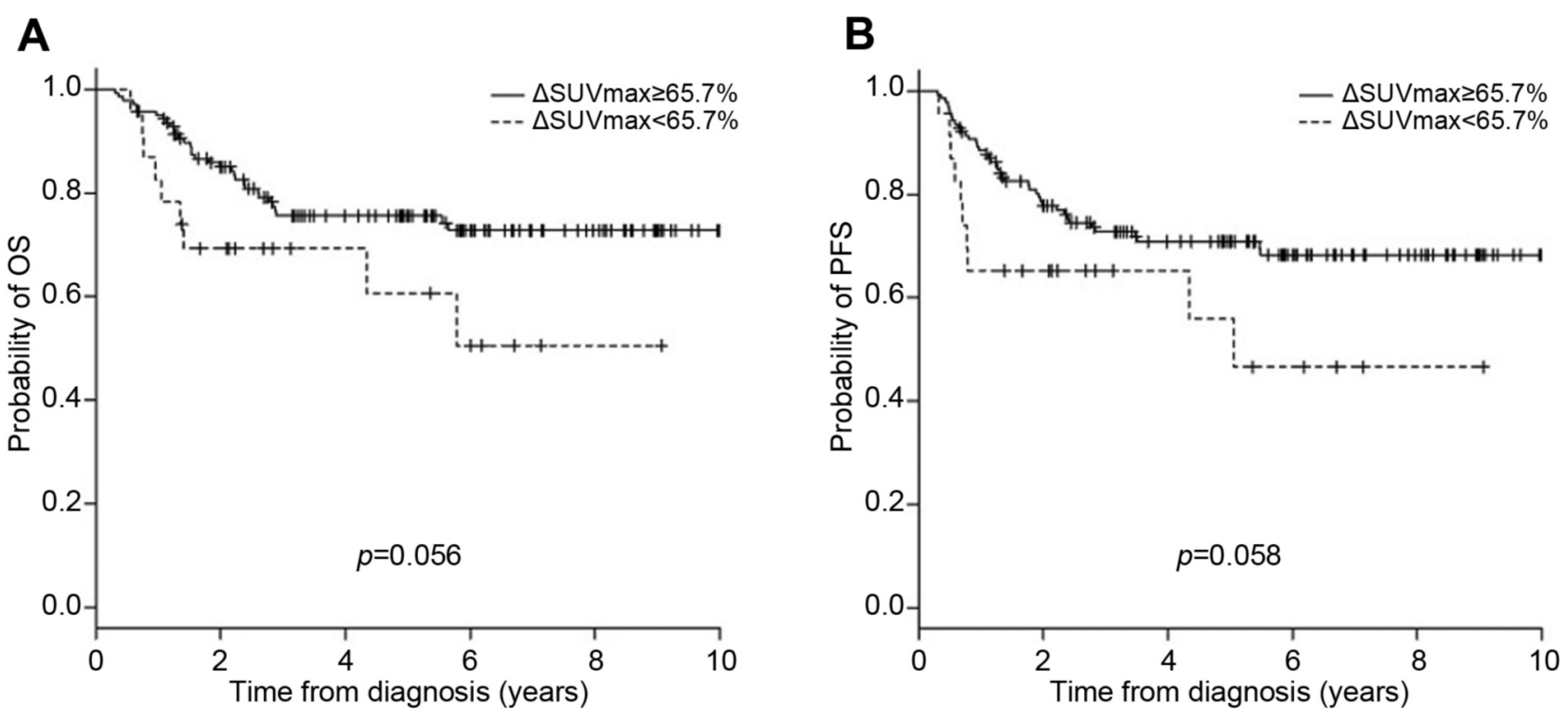

Figure 1. Kaplan-Meier survival curves for (A) overall survival (OS) and (B) progression- free survival (PFS) of all patients according to SUVbased assessment.

scans were acquired in the same anatomic locations with a $128 \times 128$ matrix and a $15.7-\mathrm{cm}$ axial FOV. The images were reconstructed using a conventional iterative algorithm (OSEM). A workstation (AW Volume Share) providing multiplanar reformatted images was also used for image display and analysis. PET/CT scans were interpreted by two nuclear medicine physicians who had no knowledge of the subject or his or her clinical information.

Visual and SUV-based assessments of iPET/CT response. Visual assessment was classified based on the Deauville 5-PS (11). Deauville scores 1-3 were considered as a good metabolic response, and scores 4-5 were considered as a residual response and progression $(26,27)$. This grading process was independent of the size of the residual tumor. Patients were assessed simultaneously based on the quantitative analysis of changes in FDG uptake using the percentage of $\triangle S U V \max$ between initial and interim PET/CT scans. The $\triangle S U V \max$ rate was calculated as follows: $\triangle \mathrm{SUV} \max$ $(\%)=100 \times[$ SUVmax (initial) - SUVmax (interim) $] /$ SUVmax (initial). For each PET dataset, the SUVmax was defined as the highest SUV among all hypermetabolic tumor foci. A $\triangle$ SUVmax value of $65.7 \%$ was used as a cutoff, based on a previous study (26).

Ethical approval. All procedures in this study that involved human participants were performed in accordance with the ethical standards of the institutional and/or national research committee and with the 1964 Declaration of Helsinki and its later amendments or comparable ethical standards. Informed consent was obtained from all individual participants included in the study.

Statistical analyses. Categorical variables were analyzed using the Chi-square test, and continuous variables were analyzed using the Student's $t$-test. Overall survival (OS) was defined as the time from diagnosis to death from any cause, and progression-free survival (PFS) was defined as the time from diagnosis to disease progression or death from any cause. The Kaplan-Meier method was used to estimate OS and PFS. Survival curves were compared using the logrank test. Prognostic factors for OS and PFS were determined by univariate and multivariate analyses using the Cox-proportional hazard model. Factors with a $p$-value of less than 0.1 in the univariate analyses were entered in the multivariate analyses. $p$-Values $<0.05$ were considered significant. For statistical analyses, SPSS version 21.0 (SPSS Inc., Chicago, IL, USA) was used.

\section{Results}

Patient characteristics. A total of 163 patients were enrolled in this study, and the median age was 61 years (range $=18-83$ years). According to the International Prognostic Index (IPI) scoring system for risk, 63 patients $(38.7 \%)$ were classified as low, 32 patients $(19.6 \%)$ as low-intermediate, 29 patients $(17.8 \%)$ as high-intermediate, and 39 patients $(23.9 \%)$ as high risk. The number of EN involvements was as follows: one site involvement in 102 patients $(62.6 \%)$, two sites in 39 patients $(23.9 \%)$, three sites in 18 patients $(11.0 \%)$, and four sites in 4 patients $(2.5 \%)$. The most common site of $\mathrm{EN}$ involvement was the stomach (35 patients, $21.5 \%$ ), and 17 patients $(10.4 \%)$ had bulky disease. Twenty-two patients $(13.5 \%)$ received autologous stem cell transplantation. Among the enrolled patients, 40 patients (23.4\%) experienced relapse. Patient characteristics are summarized in Table I.

Response evaluation of iPET/CT according to the SUV-based assessment and the Deauville 5-PS. Based on the quantitative assessment of iPET/CT using a cutoff value of $\triangle \mathrm{SUVmax}$, 140 patients $(85.9 \%)$ were classified as good responders 

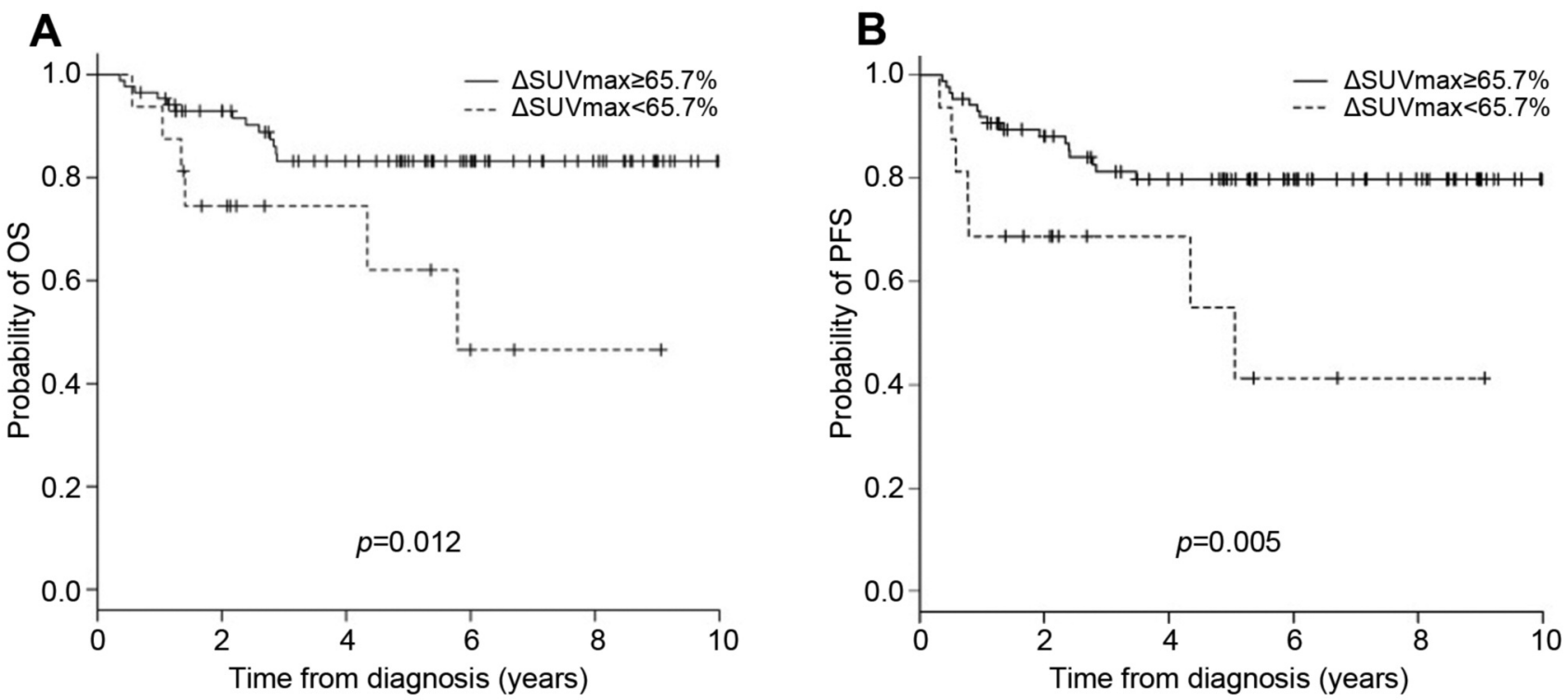

Figure 2. Kaplan-Meier survival curves for (A) overall survival (OS) and (B) progression free survival (PFS) of patients with one extranodal involvement according to SUV-based assessment.

$(\triangle \mathrm{SUVmax} \geq 65.7 \%)$ and 23 patients $(14.1 \%)$ as poor responders $(\triangle \mathrm{SUV} \max <65.7 \%)$. According to the Deauville 5-PS, 69 patients (42.3\%) were assigned a score of 1 or 2, 30 patients $(18.4 \%)$ were assigned a score of 3,57 patients $(35.0 \%)$ were assigned a score of 4 , and 7 patients $(4.3 \%)$ were assigned a score of 5 . Among the patients with one EN involvement, 84 patients $(82.4 \%)$ were classified as good responders $(\triangle \mathrm{SUV} \max \geq 65.7 \%)$ and 31 patients $(15.7 \%)$ as poor responders $(\Delta \mathrm{SUV} \max <65.7 \%)$ according to the SUVbased assessment, while 60 patients $(58.9 \%)$ were assigned Deauville 5-PS scores of 1-3, and 42 patients (41.2\%) were assigned Deauville scores of 4 or 5 , based on visual assessment.

Assessment of the iPET/CT response using the cutoff of $\triangle$ SUVmax showed a positive predictive value (PPV) of $89.1 \%$ and a negative predictive value (NPV) of $78.2 \%$, while the Deauville 5-PS (scores 1-3 vs. 4-5) showed a PPV of $91.9 \%$ and an NPV of $68.1 \%$. In patients with one EN involvement, $\triangle \mathrm{SUV}$ max showed a PPV of $83.9 \%$ and an NPV of $68.7 \%$, while the Deauville 5-PS (score 1-3 vs. 4-5) showed a PPV of $89.7 \%$ and an NPV of $66.2 \%$.

Survival outcomes. Median follow-up duration was 52.5 months (range $=3.7-119.8$ months). OS at 5 years was $80 \%$ and $63.3 \%$ for patients with one and more than one EN involvements, respectively $(p=0.005)$. PFS at 5 years was $76.3 \%$ and $56.7 \%$ in one and more than one EN site involvements, respectively $(p=0.003)$. According to the SUVbased assessment, 5-year OS rates were $75.6 \%$ and $60.6 \%$ in patients who were classified as good $(\Delta \mathrm{SUVmax} 65.7 \%)$ and poor $(\triangle \mathrm{SUVmax}<65.7 \%) \quad$ responders, respectively $(p=0.056)$. Five-year PFS rates were $77.9 \%$ and $55.9 \%$ in patients who were classified as good and poor responders, respectively ( $p=0.058$ ) (Figure 1$)$. In patients with one EN involvement, $\triangle \mathrm{SUV}$ max successfully predicted the survival outcomes in terms of OS $(83.2 \%$ vs. $62.1 \%$ at 5 years, $p=0.012)$ and PFS ( $86.9 \%$ vs. $41.3 \%$ at 5 years, $p=0.005$ ) (Figure 2). However, $\triangle \mathrm{SUV}$ max did not predict long-term survival for patients with more than one EN involvements.

According to the Deauville 5-PS, 5-year OS rates were $77.3 \%$ in patients assigned scores of 1 or $2,72.5 \%$ in patients assigned a score of $3,71.4 \%$ in patients assigned a score of 4 , and $25.0 \%$ in patients assigned a score of $5(p=0.061)$. Fiveyear PFS rates were $69.7 \%$ in patients assigned scores of 1 or $2,65.8 \%$ in patients assigned a score of 3 , and $63.4 \%$ in patients assigned a score of $4(p=0.001)$ (Figure 3$)$. There were no statistically significant survival differences between patients classified as good responders (Deauville 5-PS scores 1-3) and poor responders (Deauville 5-PS scores 4-5) in terms of OS and PFS in patients with one EN involvement and those with more than one EN involvements.

Factors affecting long-term survival. In the univariate analyses for OS, old age, Eastern Cooperative Oncology Group performance status (ECOG PS) 2-3, increased lactate dehydrogenase (LDH), EN involvement, bone marrow (BM) involvement, stage III/IV, and international prognostic index (IPI) high/high-intermediate were unfavorable factors in overall patients, while ECOG PS 2-3, BM involvement and $\triangle \mathrm{SUVmax}<65.7 \%$ in patients with one $\mathrm{EN}$ involvement 

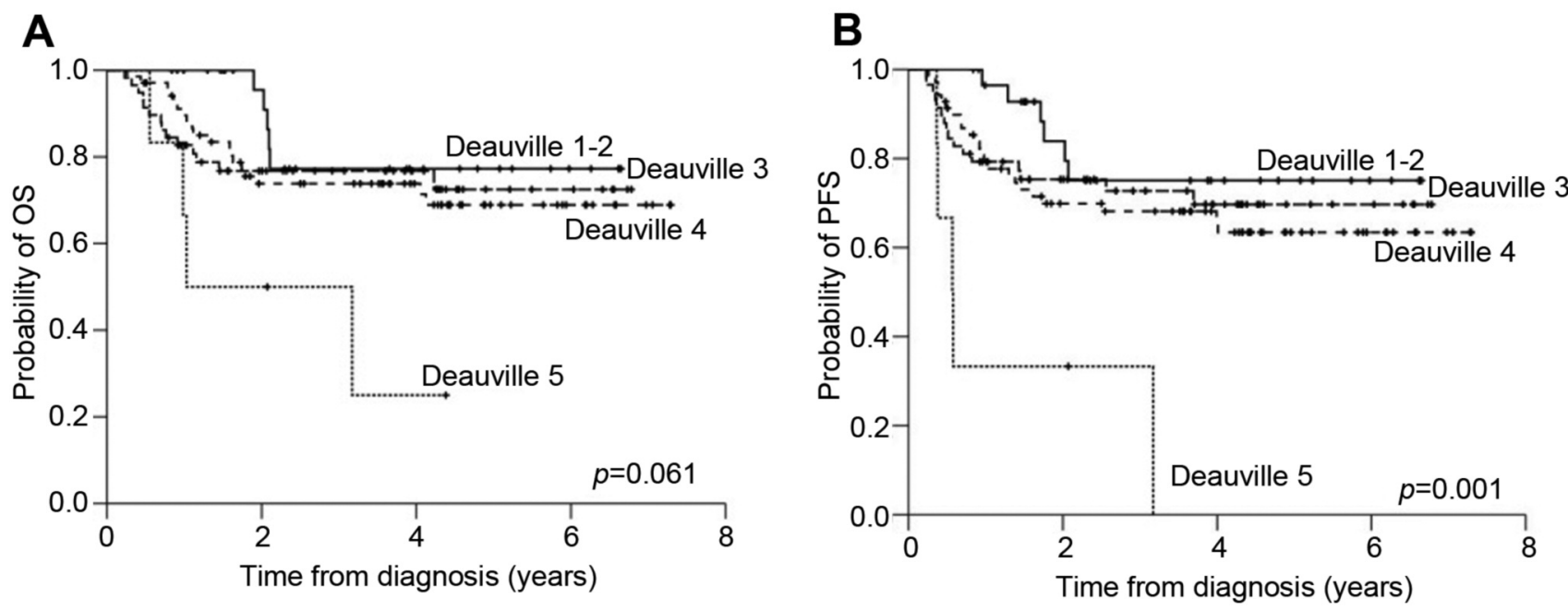

Figure 3. Kaplan-Meier survival curves for (A) overall survival (OS) and (B) progression-free survival (PFS) of all patients according to visual assessment using Deauville 5-PS.

were unfavorable factors. In the univariate analyses for PFS, old age, ECOG PS 2-3, increased LDH, EN involvement, $\mathrm{BM}$ involvement, stage III/IV, IPI high/high-intermediate, and $\triangle$ SUVmax $<65.7 \%$ were unfavorable factors in overall patients, while ECOG PS 2-3, BM involvement, and $\triangle \mathrm{SUVmax}<65.7 \%$ in patients with one $\mathrm{EN}$ involvement were unfavorable factors. In multivariate analysis, old age $(\mathrm{HR}=1.033, p=0.022)$, ECOG PS 2-3 (HR=3.525, $p<0.001)$, $\mathrm{BM}$ involvement $(\mathrm{HR}=2.972, \quad p=0.002), \quad$ and $\triangle \mathrm{SUVmax}<65.7 \%(\mathrm{HR}=2.378, p=0.034)$ were significantly associated with inferior OS rates, while ECOG PS 2-3 $(\mathrm{HR}=2.553, p=0.012), \quad \mathrm{EN}$ involvement $(\mathrm{HR}=2.370$, $p=0.008)$, and $\Delta S U V \max <65.7 \%(\mathrm{HR}=2.675, p=0.007)$ were associated with inferior PFS. Among patients with one EN involvement, ECOG PS $2-3$ and $\triangle S U V \max <65.7 \%$ were unfavorable factors for OS and PFS (Table II). Visual assessment using the Deauville 5-PS was not an independent prognostic factor in this study, regardless of the number of $\mathrm{EN}$ involvements.

\section{Discussion}

Although iPET/CT interpretation has become more standardized, the question of which methods are best for iPET/CT assessment in DLBCL with EN involvement remains controversial. The present study investigated the prognostic significance of iPET/CT by comparing SUVbased assessment and the Deauville visual assessment in DLBCL patients with EN involvement. Our results show that the SUV-based assessment is superior to the Deauville score in predicting the long-term survival outcomes for DLBCL patients with EN involvement.
In the current study, iPET/CT responses assessed with $\triangle$ SUVmax showed a PPV of $89.1 \%$ in EN DLBCL patients. These results suggest that the incidence of false-positive iPET/CT responses may be high in DLBCL patients who are treated with rituximab-containing regimens. Interestingly, some previous studies have observed a lower PPV for iPET/CT after using rituximab-containing therapy compared to the PPV for iPET/CT in the pre-rituximab era $(28,29)$. This is important since the mechanism of rituximab may involve inflammatory changes associated with recruitment of immune cells to the tumor, which likely accounts for the discrepancy in iPET/CT predictive value (30). Meanwhile, PPV with SUV-based assessment is generally known to be superior to visual assessment (31). However, our results showed the opposite. DLBCL with low baseline SUVmax is one of the pitfalls of the SUV-based method because the baseline SUVmax could be lower than the defined cutoff value, which might lead to false-positives (32). Therefore, when using SUV-based assessments, cautious interpretation is needed in patients with a low baseline SUVmax of DLBCL. In such cases, the Deauville 5-PS might be preferred as an alternative.

Although visual assessment using the Deauville 5-PS is a reliable method for end-of-treatment assessments, it showed limitations for the interpretation of iPET/CT due to the cutoff score $(22,33,34)$. Kim et al. have demonstrated that a Deauville score of 3 is not an equivalent cutoff value in the visual assessment of iPET/CT. Instead, they suggested a cutoff score of 5 for iPET/CT in patients with DLBCL (24). Similarly, Yang et al. have also noted the lack of iPET/CT interpretation with visual assessment in aggressive nonHodgkin's lymphoma with EN involvement (19). When 
Table II. Factors affecting survival outcomes in the multivariate analysis.

\begin{tabular}{|c|c|c|c|c|}
\hline \multicolumn{5}{|l|}{ Overall patients } \\
\hline & \multicolumn{2}{|c|}{ OS } & \multicolumn{2}{|c|}{ PFS } \\
\hline & $\mathrm{HR}(95 \% \mathrm{CI})$ & $p$-Value & $\mathrm{HR}(95 \% \mathrm{CI})$ & $p$-Value \\
\hline Age $\geq 65 v s .<65$ & $1.033(1.005-1.096)$ & 0.022 & - & - \\
\hline ECOG PS 2-3vs. 0-1 & $3.525(1.813-6.852)$ & $<0.001$ & $2.553(1.228-5.309)$ & 0.012 \\
\hline EN involvement $\geq 2$ sites $v s$. 1 site & & & $2.370(1.247-4.504)$ & 0.008 \\
\hline BM involve $v s$. none & $2.972(1.473-5.998)$ & 0.002 & & \\
\hline$\Delta S U V \max <65.7 \% v s . \geq 65.7 \%$ & $2.378(1.069-5.289)$ & 0.034 & $2.675(1.304-5.486)$ & 0.007 \\
\hline
\end{tabular}

Patients with one extranodal involvement

OS

\begin{tabular}{|c|c|c|c|c|}
\hline & & \\
\hline & HR $(95 \% \mathrm{CI})$ & $p$-Value & HR $(95 \% \mathrm{CI})$ & $p$-Value \\
\hline ECOG PS 2-3 vs. 0-1 & $4.692(1.655-13.304)$ & 0.004 & $6.050(1.815-20.165)$ & 0.003 \\
\hline$\Delta S U V \max <65.7 \% v s . \geq 65.7 \%$ & $3.849(1.416-10.465)$ & 0.008 & $6.979(2.463-19.777)$ & $<0.001$ \\
\hline
\end{tabular}

BM: Bone marrow; CI: confidence interval; ECOG PS: Eastern Cooperative Oncology Group performance status; EN: extranodal; HR: hazard ratio; OS: overall survival; PFS: progression-free survival; HR: hazard ratio; ECOG PS: Eastern Cooperative Oncology Group performance status; EN: extranodal; BM: bone marrow; $\triangle$ SUVmax: reduction rate of the maximum standardized uptake value.

iPET/CT responses were assessed using the Deauville score in this study, there was no significant difference between the Deauville scores of 3 and 4 in predicting response, which means that the current Deauville 5-PS is insufficient as a predictive method for iPET/CT in EN DLBCL.

The SUVmax quantitative assessment ( $\triangle \mathrm{SUV}$ max $)$ has several advantages. Because $\triangle S U V \max$ accounts for the reduction in metabolic activity of the tumor during treatment, it is relatively objective with low interobserver variability in interpretations. In addition, $\triangle S U V \max$ is easier to apply in clinical practice, and can be used with the different SUVs obtained from different scanners and individual uptake times $(35,36)$. SUV-based assessments also have drawbacks. In this study, quantitative SUV-based assessment for iPET/CT response predicted survival outcomes accurately in DLBCL patients with one EN involvement. However, it was ineffective for DLBCL patients with more than one EN involvement; one interpretation is that each additional EN site might increase false interpretations. Another possibility is that the predictive value is decreased when the baseline SUVmax is low, as mentioned above. Therefore, welldesigned studies are needed to compare visual and SUVbased methods, even though the results of many studies suggest that SUV-based methods are associated with better PPV and long-term survival outcomes $(27,37,38)$.

In the multivariate analysis, patients with $\triangle \mathrm{SUV}$ max $<65.7 \%$ showed inferior OS and PFS rates. Moreover, EN involvement was an unfavorable factor for PFS, which agrees with the results of previous studies. Unfortunately, visual assessment using the Deauville 5-PS was not an independent prognostic factor in patients with EN DLBCL. While the present study indicated significant results, our data should be interpreted cautiously due to certain limitations. First, the current study is a retrospective evaluation. Second, we could not prove each EN site through pathological confirmation. In conclusion, the quantitative SUV-based assessment for iPET/CT was a significant prognostic indicator for long-term survival outcomes, especially in patients with one EN involvement. Whereas, the Deauville 5-PS showed limitations for assessing the iPET/CT response.

\section{Conflicts of Interest}

The Authors declare that they have no conflicts of interest in regard to this study.

\section{Authors' Contributions}

J.H.M and D.H.Y designed the study; D.W.B, H.J.C, J.K, G.S, S.A, S.J and J.S.A collected the data; S.K.S, J.L, H.K, S.J, C.M.H and J.M analyzed and interpreted the data; D.W.B and J.H.M wrote the paper; J.H.M and D.H.Y contributed to the review and revision of the manuscript.

\section{Acknowledgements}

This research was supported by the Bio \& Medical Technology Development Program of the National Research Foundation (NRF) and funded by the Korean government (MSIT) (NRF2019M3E5D1A02067961). 


\section{References}

1 Perry AM, Diebold J, Nathwani BN, MacLennan KA, MullerHermelink HK, Bast M, Boilesen E, Armitage JO and Weisenburger DD: Non-Hodgkin lymphoma in the developing world: review of 4539 cases from the International NonHodgkin Lymphoma Classification Project. Haematologica 101: 1244-1250, 2016. PMID: 27354024. DOI: 10.3324/haematol. 2016.148809

2 Shankland KR, Armitage JO and Hancock BW: Non-Hodgkin lymphoma. Lancet 380: 848-857, 2012. PMID: 22835603. DOI: $10.1016 / \mathrm{S} 0140-6736(12) 60605-9$

3 Akhtar S and Maghfoor I: Rituximab plus CHOP for diffuse large-B-cell lymphoma. N Engl J Med 346: 1830-1831; author reply 1830-1831, 2002. PMID: 12050349. DOI: 10.1056/ NEJM200206063462317

4 Feugier P, Van Hoof A, Sebban C, Solal-Celigny P, Bouabdallah R, Ferme C, Christian B, Lepage E, Tilly H, Morschhauser F, Gaulard P, Salles G, Bosly A, Gisselbrecht C, Reyes F and Coiffier B: Long-term results of the R-CHOP study in the treatment of elderly patients with diffuse large B-cell lymphoma: a study by the Groupe d'Etude des Lymphomes de l'Adulte. J Clin Oncol 23: 4117-4126, 2005. PMID: 15867204. DOI: 10.1200/JCO.2005.09.131

5 Bangerter M, Moog F, Buchmann I, Kotzerke J, Griesshammer M, Hafner M, Elsner K, Frickhofen N, Reske SN and Bergmann L: Whole-body 2-[18F]-fluoro-2-deoxy-D-glucose positron emission tomography (FDG-PET) for accurate staging of Hodgkin's disease. Ann Oncol 9: 1117-1122, 1998. PMID: 9834825. DOI: 10.1023/a:1008486928190

6 Coiffier B: Positron emission tomography and gallium metabolic imaging in lymphoma. Curr Oncol Rep 3: 266-270, 2001. PMID: 11296138. DOI: 10.1007/s11912-001-0060-1

7 Cheson BD, Pfistner B, Juweid ME, Gascoyne RD, Specht L, Horning SJ, Coiffier B, Fisher RI, Hagenbeek A, Zucca E, Rosen ST, Stroobants S, Lister TA, Hoppe RT, Dreyling M, Tobinai K, Vose JM, Connors JM, Federico M, Diehl V and International Harmonization Project on L: Revised response criteria for malignant lymphoma. J Clin Oncol 25: 579-586, 2007. PMID: 17242396. DOI: $10.1200 / \mathrm{JCO} .2006 .09 .2403$

8 Terasawa T, Lau J, Bardet S, Couturier O, Hotta T, Hutchings M, Nihashi T and Nagai H: Fluorine-18-fluorodeoxyglucose positron emission tomography for interim response assessment of advanced-stage Hodgkin's lymphoma and diffuse large B-cell lymphoma: a systematic review. J Clin Oncol 27: 1906-1914, 2009. PMID: 19273713. DOI: 10.1200/JCO.2008.16.0861

9 Zhu Y, Lu J, Wei X, Song S and Huang G: The predictive value of interim and final $[18 \mathrm{~F}]$ fluorodeoxyglucose positron emission tomography after rituximab-chemotherapy in the treatment of nonHodgkin's lymphoma: a meta-analysis. Biomed Res Int 2013: 275805, 2013. PMID: 24288671. DOI: $10.1155 / 2013 / 275805$

10 Adams HJ and Kwee TC: Prognostic value of interim FDGPET in R-CHOP-treated diffuse large B-cell lymphoma: Systematic review and meta-analysis. Crit Rev Oncol Hematol 106: 55-63, 2016. PMID: 27637352. DOI: 10.1016/j.critrevonc. 2016.07.003

11 Meignan M, Gallamini A, Meignan M, Gallamini A and Haioun C: Report on the first International Workshop on interim-PETscan in lymphoma. Leuk Lymphoma 50: 1257-1260, 2009. PMID: 19544140 . DOI: 10.1080/10428190903040048
12 Itti E, Juweid ME, Haioun C, Yeddes I, Hamza-Maaloul F, El Bez I, Evangelista E, Lin C, Dupuis J and Meignan M: Improvement of early $18 \mathrm{~F}-\mathrm{FDG}$ PET interpretation in diffuse large B-cell lymphoma: importance of the reference background. J Nucl Med 51: 1857-1862, 2010. PMID: 21078789. DOI: 10.2967/jnumed.110.080556

13 Michallet AS, Trotman J and Tychyj-Pinel C: Role of early PET in the management of diffuse large B-cell lymphoma. Curr Opin Oncol 22: 414-418, 2010. PMID: 20683268. DOI: 10.1097/ CCO.0b013e32833d5905

14 International Non-Hodgkin's Lymphoma Prognostic Factors Project: A predictive model for aggressive non-Hodgkin's lymphoma. N Engl J Med 329: 987-994, 1993. PMID: 8141877. DOI: 10.1056/NEJM199309303291402

15 Sehn LH, Berry B, Chhanabhai M, Fitzgerald C, Gill K, Hoskins P, Klasa R, Savage KJ, Shenkier T, Sutherland J, Gascoyne RD and Connors JM: The revised International Prognostic Index (RIPI) is a better predictor of outcome than the standard IPI for patients with diffuse large B-cell lymphoma treated with RCHOP. Blood 109: 1857-1861, 2007. PMID: 17105812. DOI: 10.1182/blood-2006-08-038257

16 Hutchings M, Loft A, Hansen M, Pedersen LM, Berthelsen AK, Keiding S, D'Amore F, Boesen AM, Roemer L and Specht L: Position emission tomography with or without computed tomography in the primary staging of Hodgkin's lymphoma. Haematologica 91: 482-489, 2006. PMID: 16585015.

17 Khan AB, Barrington SF, Mikhaeel NG, Hunt AA, Cameron L, Morris T and Carr R: PET-CT staging of DLBCL accurately identifies and provides new insight into the clinical significance of bone marrow involvement. Blood 122: 61-67, 2013. PMID: 23660958. DOI: 10.1182/blood-2012-12-473389

18 Barrington SF and O'Doherty MJ: Limitations of PET for imaging lymphoma. Eur J Nucl Med Mol Imaging 30: S117-127, 2003. PMID: 12748831. DOI: 10.1007/s00259-003-1169-2

19 Yang DH, Ahn JS, Byun BH, Min JJ, Kweon SS, Chae YS, Sohn SK, Lee SW, Kim HW, Jung SH, Kim YK, Kim HJ, Bom HS and Lee JJ: Interim PET/CT-based prognostic model for the treatment of diffuse large B cell lymphoma in the post-rituximab era. Ann Hematol 92: 471-479, 2013. PMID: 23238895. DOI: 10.1007/s00277-012-1640-x

20 Moskowitz CH, Schoder H, Teruya-Feldstein J, Sima C, Iasonos A, Portlock CS, Straus D, Noy A, Palomba ML, O'Connor OA, Horwitz S, Weaver SA, Meikle JL, Filippa DA, Caravelli JF, Hamlin PA and Zelenetz AD: Risk-adapted dose-dense immunochemotherapy determined by interim FDG-PET in Advanced-stage diffuse large B-Cell lymphoma. J Clin Oncol 28: 1896-1903, 2010. PMID: 20212248. DOI: 10.1200/ JCO.2009.26.5942

21 Han HS, Escalon MP, Hsiao B, Serafini A and Lossos IS: High incidence of false-positive PET scans in patients with aggressive non-Hodgkin's lymphoma treated with rituximab-containing regimens. Ann Oncol 20: 309-318, 2009. PMID: 18842613. DOI: $10.1093 /$ annonc/mdn629

22 Pregno P, Chiappella A, Bello M, Botto B, Ferrero S, Franceschetti S, Giunta F, Ladetto M, Limerutti G, Menga M, Nicolosi M, Priolo G, Puccini B, Rigacci L, Salvi F, Vaggelli L, Passera R, Bisi G and Vitolo U: Interim 18-FDG-PET/CT failed to predict the outcome in diffuse large B-cell lymphoma patients treated at the diagnosis with rituximab-CHOP. Blood 119: 2066-2073, 2012. PMID: 22234681. DOI: 10.1182/blood-2011-06-359943 
23 Yoo C, Lee DH, Kim JE, Jo J, Yoon DH, Sohn BS, Kim SW, Lee JS and Suh C: Limited role of interim PET/CT in patients with diffuse large B-cell lymphoma treated with R-CHOP. Ann Hematol 90: 797-802, 2011. PMID: 21181163. DOI: 10.1007/ s00277-010-1135-6

24 Kim J, Song YS, Lee JS, Lee WW and Kim SE: Risk stratification of diffuse large B-cell lymphoma with interim PETCT based on different cutoff Deauville scores. Leuk Lymphoma 59: 340-347, 2018. PMID: 28629257. DOI: 10.1080/ 10428194.2017.1339877

25 Coiffier B, Lepage E, Briere J, Herbrecht R, Tilly H, Bouabdallah R, Morel P, Van Den Neste E, Salles G, Gaulard P, Reyes F, Lederlin P and Gisselbrecht C: CHOP chemotherapy plus rituximab compared with CHOP alone in elderly patients with diffuse large-B-cell lymphoma. N Engl J Med 346: 235242, 2002. PMID: 11807147. DOI: 10.1056/NEJMoa011795

26 Meignan M, Gallamini A, Haioun C and Polliack A: Report on the Second International Workshop on interim positron emission tomography in lymphoma held in Menton, France, 8-9 April 2010. Leuk Lymphoma 51: 2171-2180, 2010. PMID: 21077737. DOI: $10.3109 / 10428194.2010 .529208$

27 Lin C, Itti E, Haioun C, Petegnief Y, Luciani A, Dupuis J, Paone G, Talbot JN, Rahmouni A and Meignan M: Early 18F-FDG PET for prediction of prognosis in patients with diffuse large Bcell lymphoma: SUV-based assessment versus visual analysis. J Nucl Med 48: 1626-1632, 2007. PMID: 17873129. DOI: 10.2967/jnumed.107.042093

28 Juweid ME, Wiseman GA, Vose JM, Ritchie JM, Menda Y, Wooldridge JE, Mottaghy FM, Rohren EM, Blumstein NM, Stolpen A, Link BK, Reske SN and Graham MM and Cheson BD: Response assessment of aggressive non-Hodgkin's lymphoma by integrated International Workshop Criteria and fluorine-18-fluorodeoxyglucose positron emission tomography. J Clin Oncol 23: 4652-4661, 2005. PMID: 15837965. DOI: 10.1200/JCO.2005.01.891

29 Haioun C, Itti E, Rahmouni A, Brice P, Rain JD, Belhadj K, Gaulard P, Garderet L, Lepage E, Reyes F and Meignan M: [18F]fluoro-2-deoxy-D-glucose positron emission tomography (FDG-PET) in aggressive lymphoma: an early prognostic tool for predicting patient outcome. Blood 106: 1376-1381, 2005. PMID: 15860666. DOI: 10.1182/blood-2005-01-0272

30 Spaepen K, Stroobants S, Dupont P, Bormans G, Balzarini J, Verhoef G, Mortelmans L, Vandenberghe P and De Wolf-Peeters C: [(18)F]FDG PET monitoring of tumour response to chemotherapy: does [(18)F]FDG uptake correlate with the viable tumour cell fraction? Eur J Nucl Med Mol Imaging 30: 682-688, 2003. PMID: 12601498. DOI: 10.1007/s00259-003-1120-6

31 Le Gouill S and Casasnovas RO: Interim PET-driven strategy in de novo diffuse large B-cell lymphoma: do we trust the driver? Blood 129: 3059-3070, 2017. PMID: 28416502. DOI: 10.1182/ blood-2016-05-672196
32 Casasnovas RO, Meignan M, Berriolo-Riedinger A, Bardet S, Julian A, Thieblemont C, Vera P, Bologna S, Briere J, Jais JP, Haioun C, Coiffier B, Morschhauser F and Groupe d'etude des lymphomes de la: SUVmax reduction improves early prognosis value of interim positron emission tomography scans in diffuse large B-cell lymphoma. Blood 118: 37-43, 2011. PMID: 21518924. DOI: 10.1182/blood-2010-12-327767

33 Carr R, Fanti S, Paez D, Cerci J, Gyorke T, Redondo F, Morris TP, Meneghetti C, Auewarakul C, Nair R, Gorospe C, Chung JK, Kuzu I, Celli M, Gujral S, Padua RA, Dondi M and Group ILS: Prospective international cohort study demonstrates inability of interim PET to predict treatment failure in diffuse large B-cell lymphoma. J Nucl Med 55: 1936-1944, 2014. PMID: 25429159. DOI: $10.2967 /$ jnumed.114.145326

34 Mamot C, Klingbiel D, Hitz F, Renner C, Pabst T, Driessen C, Mey U, Pless M, Bargetzi M, Krasniqi F, Gigli F, Hany T, Samarin A, Biaggi C, Rusterholz C, Dirnhofer S, Zucca E and Martinelli G: Final results of a prospective evaluation of the predictive value of interim positron emission tomography in patients with diffuse large B-cell lymphoma treated with RCHOP-14 (SAKK 38/07). J Clin Oncol 33: 2523-2529, 2015. PMID: 26150440. DOI: 10.1200/JCO.2014.58.9846

35 Casasnovas RO, Ysebaert L, Thieblemont C, Bachy E, Feugier P, Delmer A, Tricot S, Gabarre J, Andre M, Fruchart C, Mounier N, Delarue R, Meignan M, Berriolo-Riedinger A, Bardet S, Emile JF, Jais JP, Haioun C, Tilly H and Morschhauser F: FDGPET-driven consolidation strategy in diffuse large B-cell lymphoma: final results of a randomized phase 2 study. Blood 130: 1315-1326, 2017. PMID: 28701367. DOI: 10.1182/blood2017-02-766691

36 Kasamon YL and Wahl RL: FDG PET and risk-adapted therapy in Hodgkin's and non-Hodgkin's lymphoma. Curr Opin Oncol 20: 206-219, 2008. PMID: 18300772. DOI: 10.1097/CCO. 0b013e3282f5123d

37 Itti E, Meignan M, Berriolo-Riedinger A, Biggi A, Cashen AF, Vera P, Tilly H, Siegel BA, Gallamini A, Casasnovas RO and Haioun C: An international confirmatory study of the prognostic value of early PET/CT in diffuse large B-cell lymphoma: comparison between Deauville criteria and DeltaSUVmax. Eur J Nucl Med Mol Imaging 40: 1312-1320, 2013. PMID: 23649463. DOI: 10.1007/s00259-013-2435-6

38 Itti E, Lin C, Dupuis J, Paone G, Capacchione D, Rahmouni A, Haioun C and Meignan M: Prognostic value of interim 18F-FDG PET in patients with diffuse large B-Cell lymphoma: SUV-based assessment at 4 cycles of chemotherapy. J Nucl Med 50: 527 533, 2009. PMID: 19289424. DOI: 10.2967/jnumed.108.057703

Received March 26, 2020

Revised April 1, 2020

Accepted April 2, 2020 